\title{
Prognostic Significance of Targeted Magnetic Resonance Coronary Angiography to Predict Cardiac Events in Patients with Known or Suspected Coronary Artery Disease
}

\author{
Yodying Kaolawanich \\ Mahidol University \\ Rawiwan Thongsongsang \\ Mahidol University \\ Thananya Boonyasirinant ( $\square$ drthananyaa@yahoo.com ) \\ Mahidol University
}

\section{Research Article}

Keywords: cardiac magnetic resonance imaging, coronary artery disease, magnetic resonance coronary angiography, prognosis

Posted Date: May 14th, 2021

DOl: https://doi.org/10.21203/rs.3.rs-478379/v1

License: (c) (1) This work is licensed under a Creative Commons Attribution 4.0 International License.

Read Full License 


\section{Abstract}

Background Magnetic resonance coronary angiography (MRCA) is one of the most promising tools for noninvasive imaging of coronary arteries without radiation exposure or contrast media administration. However, knowledge about the prognostic value of MRCA is limited.

Methods The study included 389 patients with known or suspected coronary artery disease (CAD) who underwent clinical cardiac magnetic resonance (CMR) including MRCA imaging. The presence of significant coronary artery stenosis was defined by visual estimation of $\geq 50 \%$ diameter reduction using targeted MRCA images. Patients were followed up for hard cardiac events (cardiac death or non-fatal myocardial infarction) and major adverse cardiac events (MACE) that also included hospitalization for heart failure and late revascularization (>180 days after the CMR study).

Results Average age was $68 \pm 11$ years and $48 \%$ were male. One hundred and thirty-nine patients had significant stenosis on MRCA. During a median follow-up period of 53.9 months, 23 hard cardiac events and 52 MACE occurred. Patients with significant coronary artery stenosis had higher rates of hard cardiac events (annual event rate, $3.12 \%$ versus $0.56 \%$; HR 5.52; $95 \% \mathrm{Cl} 2.17-14.01 ; \mathrm{p}<0.001$ ) and MACE (annual event rate, $6.44 \%$ vs. $1.83 \%$; HR 3.49; $95 \% \mathrm{Cl} 1.98-6.14$; $p<0.001$ ) than those without significant stenosis. Multivariate analyses identified significant coronary artery stenosis as an independent predictor of hard cardiac events (HR 3.35; 95\% Cl 1.13-9.96; $p=0.03$ ) and MACE (HR 2.00; 95\% Cl 1.02-3.90; $p=0.04)$. MRCA presented an incremental prognostic value over clinical factors, left ventricular ejection fraction, and myocardial scarring to predict hard cardiac events $(p=0.03)$.

Conclusion Targeted MRCA demonstrated independent and incremental prognostic values to predict future cardiac events in patients with known or suspected CAD.

\section{Introduction}

Coronary artery disease (CAD) is one of the leading causes of morbidity and mortality worldwide. ${ }^{[1]}$ Diagnosis and risk stratification are crucial elements in the management of CAD. Assessment of coronary artery stenosis is also an important part of comprehensive CAD evaluation. Coronary computed tomography angiography (CCTA) has rapidly emerged as a noninvasive method that provides visualization of the coronary arteries and detection of luminal narrowing. CCTA has excellent diagnostic accuracy as well as providing prognostic value in patients with known or suspected CAD. ${ }^{[2-3]}$ However, several factors are known to deteriorate image quality and interpretation including severe calcification

and elevated heart rate. ${ }^{[4-6]}$ Moreover, radiation exposure and the administration of contrast media are recognized limitations of CCTA. Magnetic resonance coronary angiography (MRCA) is currently one of the most promising techniques for noninvasive imaging of coronary arteries. MRCA provides high sensitivity and specificity in the diagnosis of CAD without radiation exposure or contrast media administration. ${ }^{[7-9]}$ 
Whole-heart and targeted approaches are the two main acquisition methods for MRCA. The whole-heart technique is mostly performed for visualization of all three major coronary arteries and offers a relatively reduced total examination period. However, the targeted approach provides details of each coronary artery and is more likely to yield higher image quality as well as vessel sharpness. ${ }^{[10]}$

Several studies have addressed the diagnostic accuracy of MRCA, including a recent meta-analysis of more than 20 studies. ${ }^{[7]}$ By contrast, the prognostic data of MRCA is very limited. One study demonstrated the prognostic significance of whole-heart MRCA to predict cardiac events in patients with suspected CAD. ${ }^{[11]}$ However, sample numbers were relatively small $(n=207)$, and no data were provided on left ventricular (LV) function or late gadolinium enhancement (LGE), which are fundamental parts of routine clinical cardiac magnetic resonance (CMR). ${ }^{[11]}$

In this study, we sought to determine the prognostic value of targeted MRCA combining routine clinical CMR in a larger population of patients with known or suspected CAD.

\section{Methods}

\section{Study Population}

Consecutive patients over 18 years of age referred for clinical CMR including MRCA imaging were enrolled between 2010 and 2015. MRCA was performed as a part of our comprehensive CMR protocol for the evaluation of patients with CAD. Detailed medical history was collected on the day of the CMR examination. History of hypertension, diabetes mellitus, hyperlipidemia, CAD, and stroke were defined by recent guidelines. ${ }^{[12-15]}$

Patients were excluded from the study if they had poor MRCA images or their follow-up data could not be obtained. The study was done in accordance with the Declaration of Helsinki. The institutional ethics committee (Siriraj Institutional Review Board [SIRB], Faculty of Medicine Siriraj Hospital, Mahidol University) approved this retrospective study and waived the need for additional written informed consent.

\section{CMR Protocol}

CMR was performed to assess cardiac function, coronary arteries, and LGE on a 1.5 Tesla Gyroscan NT Philips Scanner (Philips Medical Systems, Best, The Netherlands). Functional study and LGE images were acquired as previously described. ${ }^{[16]}$

\section{MRCA Imaging [17]}

Non-contrast, free-breathing, targeted MRCA scanning was performed with a 32-element cardiac synergy receiver coil. An electrocardiogram (ECG) was synchronized during scanning by a vectorcardiogram using three electrodes placed on the chest wall. ${ }^{[18]}$ Imaging of the coronary arteries was acquired in the mid- 
diastolic phase of the cardiac cycle. Respiratory motion compensation was performed using the navigator technique by placing a navigator on the right hemidiaphragm. ${ }^{[19]}$

In the process of coronary artery acquisition, a multi-stack survey was first acquired to provide the location of the navigator and the position of low-resolution MRCA. Multiphase cine images were then performed to determine a subject-specific trigger delay and proper acquisition window during minimal cardiac motion. Next, low-resolution MRCA was acquired by planning the slice in the transverse plane, covering the whole heart from the base to apex. The images from this sequence were used to plan double oblique three-dimensional (D) volume for the right coronary artery (RCA) using the three-point plan-scan tool. The corresponding image plan was then applied to acquire high-resolution 3-D MRCA using spectral pre-saturation with inversion recovery (SPIR).

For coronary artery localization and navigator positioning at the right hemidiaphragm, ECG triggered, freebreathing, multi-stack 2-D segmented gradient echo scans from thoracic transverse, sagittal and coronal images were applied to identify the positions of the heart and diaphragm.

To determine the motion of the coronary arteries, multiphase cine images were obtained in the transverse plane perpendicular to the RCA, using a steady-state free precession (SSFP) pulse sequence. ${ }^{20]}$ Parameters for MRCA acquisition were echo time: 1.63 milliseconds (ms), repetition time: $3.3 \mathrm{~ms}$, flip angle: 60 degrees, matrix size: $200 \times 172$, number of cardiac phases: 80 and acceleration factor (SENSE): 2.0. The motion of the RCA was visually assessed by scrolling through the transverse cine images. [20] The rest period of minimal displacement during the diastole period was defined as trigger delay time and optimal acquisition window. These timings were then used for subsequent 3-D coronary acquisitions. ${ }^{\text {[20] }}$

\section{Image Analysis}

LV volumes and ejection fraction (EF) were quantitatively measured from the stack of short-axis cine images using standard techniques. ${ }^{[16]}$ The presence of hyperenhanced tissue on LGE images, interpreted as representing myocardial scarring, was determined by visual inspection using the American Heart Association (AHA) 17-segment model. [21]

For MRCA images, epicardial coronary artery segmentation was determined according to a modified AHA classification. ${ }^{[22]}$ Similar to the previous investigation, the presence of significant coronary lesions was determined based on visual estimation ( $\geq 50 \%$ diameter reduction). ${ }^{[11]}$

\section{Clinical Follow-Up}

Follow-up data were collected from clinical visits and medical records. Patients were followed up for hard cardiac events and major adverse cardiac events (MACE). Hard cardiac events were defined by the composite outcomes of cardiac death and non-fatal MI, while MACE was defined as the composite outcomes of cardiac death, non-fatal Ml, hospitalization for heart failure, and late coronary 
revascularization (revascularization procedures that occurred more than 180 days after the CMR study). [23]

CMR results may influence decisions regarding revascularization and lead to periprocedural events or death. Therefore, coronary revascularizations that occurred within 180 days after the CMR study or periprocedural events that occurred in the same admission were not considered for analysis.

\section{Statistical Analysis}

Statistical analyses were performed using IBM SPSS Statistics for Windows, version 20.0 (IBM Corp., Armonk, NY, USA). Continuous variables with normal distribution were presented as mean \pm standard deviation (SD), and continuous variables with non-normal distribution were presented as median and interquartile ranges. The normality of the distribution of variables was examined by the KolmogorovSmirnov test. Categorical variables were present as absolute numbers and percentages. Differences between patients with and without significant stenosis in terms of clinical baseline and CMR characteristics were compared using the Student's unpaired t-test or the Mann-Whitney U test for continuous variables, while the chi-square test or Fisher's exact test were used for categorical variables, as appropriate.

Composite outcomes between patients with and without significant stenosis were estimated using the Kaplan-Meier method and compared with the log-rank test. To analyze the predictors of hard cardiac events and MACE, a Cox-regression analysis was performed to assess univariate predictors from baseline characteristics, medications, and CMR parameters. The five most significant predictors identified by the univariate analysis were included as candidate variables in a stepwise selection process. To avoid the potential for overfitting, no more than five variables were included in any multivariate model.

To assess the incremental prognosis values of significant predictors, global chi-square values were calculated after adding predictors in the following order: clinical factors, LVEF, LGE, and MRCA.

The hazard ratios (HRs) and 95\% confidence intervals (Cls) of the outcomes were calculated, with a pvalue $<0.05$ considered statistically significant.

\section{Results}

\section{Patient Characteristics}

Three hundred and ninety-seven patients were enrolled, 8 were excluded: 6 had uninterpretable MRCA images, and 2 did not have adequate follow-up data. Thus, 389 patients were included for the final analysis. Baseline characteristics of patients with and without significant coronary artery stenosis are listed in Table 1. Average age was $68.1 \pm 10.8$ years, and $48 \%$ were male. The common presenting symptoms were chest pain (34\%), dyspnea (16\%), and heart failure syndrome. Sixty-one patients had a history of stable CAD, and 12 patients had previous MI. 
One hundred and thirty-nine patients had significant stenosis of at least one segment of a coronary artery, while 250 patients were free from significant stenosis. Among the 139 patients with significant stenosis, 66 had single vessel disease, while 73 had multivessel disease. Patients with significant coronary artery stenosis were more likely to have hyperlipidemia, stable CAD, previous $\mathrm{Ml}$, and be on antiplatelet and statin therapy ( $p<0.05$ for all). Patients with significant coronary artery stenosis also had lower LVEF and higher prevalence of LGE ( $p<0.001$ for both). Figure 1 demonstrates MRCA findings in a patient with significant coronary artery stenosis.

\section{Follow-Up Results}

During a median follow-up period of $53.9(33.8,67.9)$ months, 23 hard cardiac events and 52 MACE occurred. Patients with significant coronary artery stenosis had higher rates of both hard cardiac events (annual event rate, $3.12 \%$ vs. $0.56 \%$; HR 5.52; $95 \% \mathrm{Cl} 2.17-14.01$; $p<0.001$ ) and MACE (annual event rate, $6.44 \%$ vs. $1.83 \%$; HR $3.49 ; 95 \% \mathrm{Cl} 1.98-6.14 ; p<0.001$ ) than those without significant stenosis. Table 2 shows cardiac events during follow-up. Kaplan-Meier survival curves for hard cardiac events and MACE are shown in Figure 2.

Univariate and multivariate analyses of predictors for hard cardiac events and MACE are shown in Tables $\mathbf{3}$ and $\mathbf{4}$, respectively. The five most significant predictors identified by univariate analysis for hard cardiac events were previous $\mathrm{MI}$, the use of nitrate, LVEF, LGE, and significant coronary artery stenosis on MRCA $(p<0.05$ for all). History of heart failure, previous MI, LVEF, LGE, and MRCA were the five most significant predictors for MACE ( $\mathrm{p}<0.01$ for all). Multivariate analyses showed that history of heart failure (HR 3.28; 95\% Cl 1.62-6.63; $p=0.001$ ), LGE (HR 2.27; 95\% Cl 1.05-4.91; $p=0.03$ ) and MRCA (HR 2.00; 95\% Cl 1.023.90; $p=0.04$ ) were independent predictors of MACE (Table 4). For hard cardiac events, only significant coronary artery stenosis on MRCA was an independent predictor (HR 3.55; 95\% Cl 1.13-9.96; $p=0.03$ ) (Table 3).

Figure 3 shows incremental prognostic values of clinical and CMR variables to predict hard cardiac events and MACE. When the prognosis was assessed in a hierarchical manner (clinical only, clinical+LVEF, clinical+LVEF+LGE, and clinical+LVEF+LGE+MRCA), MRCA provided an incremental prognostic value over clinical variables, LVEF, and LGE for hard cardiac events $(p=0.03)$ (Figure 3A) but did not reach a significance for MACE $(p=0.17)$ (Figure 3B).

In this study, targeted MRCA also detected anomalous coronary arteries; two patients had the RCA originated from the left coronary cusp of the aortic valve, while one of these had a malignant type, interarterial course with an acute angle requiring surgical managements (Figure 4).

\section{Discussion}

Result showed that significant stenosis of the coronary artery assessed by targeted MRCA was a strong and independent predictor of hard cardiac events and MACE in patients with known or suspected CAD. 
Targeted MRCA also provided an incremental prognostic value for hard cardiac events over clinical data, LVEF, and myocardial scarring.

\section{The Use of CMR in Patients with CAD}

Many studies have been published regarding the role of CMR in CAD over the last decade providing important technical advances, large-scale clinical validation and prognostic data. ${ }^{[24,25]}$ CMR can evaluate global and regional cardiac function, myocardial ischemia and myocardial scarring in one examination. The standard CMR protocol for evaluation of CAD comprises cardiac function, myocardial perfusion and LGE imaging. Coronary artery assessment by MRCA is a novel sequence that can provide additional diagnostic and prognostic value. ${ }^{[7-9]}$

\section{Assessment of Coronary Arteries using MRCA}

MRCA is more technically challenging than CMR of other vascular beds due to several unique issues including small luminal size and near constant motion of coronary arteries, the high level of tortuosity, and surrounding signals from adjacent epicardial fat and myocardium. According to a recent guideline, current roles of MRCA include the evaluation of anomalous coronary arteries and coronary artery aneurysms. ${ }^{[26]}$ For atherosclerotic disease, CCTA appears to be the standard technique for noninvasive visualization of coronary arteries. ${ }^{[26]}$ Currently, the CCTA technique offers a sensitive and specific tool for the detection of significant coronary stenosis, and also plays a prognostic role in the prediction of cardiac events. ${ }^{[2,3]}$ However, exposure to ionizing radiation and contrast agent administration present inherent disadvantages. CTTA use is also limited for patients with heavy coronary artery calcification or high heart rates. ${ }^{[4-6]}$

MRCA has some benefits over CCTA. MRCA can assess coronary arteries without exposure to radiation or administration of contrast media. MRCA sequences have undergone considerable technical improvements over time. ${ }^{[27]}$ A non-contrast enhanced MRCA technique using a 3-D approach is the current standard CMR protocol due to its higher spatial resolution compared to the previous 2-D technique. ${ }^{[27]}$ Respiratory motion can be overcome by multiple breath-hold or preferably a free-breathing method, with a respiratory navigator gating.

Whole-heart and targeted approaches are the main acquisition modes used at present. Imaging of major coronary arteries by targeted MRCA is performed with several oblique, thin-slab volumes targeting specific coronary arteries, while the whole-heart approach is an alternative method that can visualize all coronary arteries within a single acquisition. A meta-analysis showed that the whole-heart technique had higher specificity (78\% versus $57 \%$ ) with similar sensitivity (89\% versus $90 \%$ ) to detect coronary artery stenosis compared to the targeted technique. ${ }^{[7]}$ However, in this meta-analysis, almost all studies of targeted MRCA (8 of 9 studies) used gradient echo sequence, whereas most studies of whole-heart MRCA (12 of 15) used SSFP sequence. Sequence type affects the accuracy of coronary artery assessment. SSFP sequence provides higher blood signal intensity, reduced motion artifacts, and superior vessel 
sharpness that can be further enhanced when used with an optimized k-space sampling strategy such as radial sampling. ${ }^{[28,29]}$

Although the whole-heart method is convenient to perform and requires less total examination time, targeted MRCA is more likely to yield high image quality and vessel sharpness, with relatively shorter acquisition time for imaging a single specific coronary artery as well as providing greater contrast-tonoise ratio. ${ }^{[10]}$ Overall, both methods have their own advantages, and could potentially be useful for clinical applications of coronary artery assessment. Our center has previously reported on the high accuracy of targeted MRCA to diagnose coronary artery stenosis compared to invasive coronary angiography with a sensitivity of $97.6 \%$ and a specificity of $75 \%$. ${ }^{[30]}$

\section{Prognostic Value of MRCA in Patients with CAD}

Unlike diagnostic data, evidence concerning the prognosis of MRCA is very limited. The study of 207 patients by Yoon et al. found that the presence of significant coronary artery stenosis using whole-heart MRCA was strongly associated with cardiac death and MACE based on univariate analysis. ${ }^{[1]}$ However, a small number of cardiac events in the study precluded a valid multivariate analysis for comparison of MRCA with other risk predictors. Given the lack of prognostic studies regarding targeted MRCA, our study was conducted based on the hypothesis that the targeted approach may have a prognostic role similar to the whole-heart technique. Additionally, we included MRCA into a routine clinical CMR protocol (functional study, LGE) to evaluate whether MRCA provides independent and incremental prognostic values.

Our study determined that $35.7 \%$ of patients who underwent MRCA for assessment of CAD had significant coronary artery stenosis. The moderate prevalence of significant coronary artery stenosis in our study was comparable with previous reports. ${ }^{[11,31]}$ Patients with significant coronary artery stenosis had a higher prevalence of cardiac risk factors such as hyperlipidemia and current smoking. They were also more likely to have myocardial scarring. Concurring with Yoon et al., our data showed that patients with significant coronary artery stenosis had a substantially higher rate of hard cardiac events and MACE than those without significant stenosis. ${ }^{[11]}$ Targeted MRCA also demonstrated an independent and incremental prognostic value over clinical data, LVEF, and myocardial scarring to predict future cardiac events. This was a novel finding to show the advantages of MRCA in patients with CAD.

In our study, targeted MRCA had another benefit beyond the assessment of atherosclerotic CAD. MRCA was able to diagnose patients who had anomalous coronary arteries. This is a unique advantage of MRCA, specifically for children and young patients who need to avoid radiation exposure, while some also need serial CMR examinations.

Novel MRCA techniques to increase spatial resolution and decrease acquisition time are currently being developed, including 4-D whole-heart imaging and accelerated acquisition methods such as compressed sensing. These techniques may improve the accuracy and prognostic significance of MRCA. 


\section{Study Limitations}

Our study had some limitations. Firstly, the study methodology was retrospective, and some confounding factors could not be completely eliminated. Secondly, our study had a relatively low event rate, and some degree of overfitting may have occurred in the multivariate analyses. This possibility was avoided by using a limited number of variables, and our results were consistent with a previous study. ${ }^{[11]}$ Thirdly, imaging of coronary stents and in-stent restenosis cannot be assessed directly by conventional MRCA because of local signal voids of the stents. Thus, evidence of MRCA in this population was limited.

Finally, the prognostic value of MRCA when combined with a comprehensive CMR study including stress perfusion imaging remains unknown and requires further study.

\section{Conclusion}

This study demonstrated the prognostic value of significant coronary artery stenosis by non-contrast enhanced targeted MRCA in a larger group of patients with known or suspected CAD. MRCA may become an integrative part of comprehensive CMR assessment of CAD in the future.

\section{List Of Abbreviations}

$\mathrm{Cl}=$ confidence interval

$\mathrm{CAD}=$ coronary artery disease

CCTA = coronary computed tomography angiography

$\mathrm{CMR}=$ cardiac magnetic resonance

ECG $=$ electrocardiogram

$\mathrm{HR}=$ hazard ratio

$\mathrm{LGE}=$ late gadolinium enhancement

$\mathrm{LV}=$ left ventricular

$L V E F=$ left ventricular ejection fraction

MACE = major adverse cardiovascular events

$\mathrm{MI}=$ myocardial infarction

MRCA = magnetic resonance coronary angiography

SSFP = steady state free precession 


\section{Declarations}

\section{Ethics approval and consent to participate}

This study was approved by Siriraj Institutional Review Board (SIRB), Faculty of Medicine Siriraj Hospital, Mahidol University (Title: Prognostic value of comprehensive cardiac magnetic resonance imaging in patients with suspected coronary artery disease, certification of approval number: Si 175/2014). All methods involving human data were performed in accordance with the Declaration of Helsinki. The need for consent was waived by Siriraj Institutional Review Board (SIRB) due to its retrospective nature and as all personal identifying information was obliterated.

\section{Consent for publication}

Not applicable.

\section{Availability of data and material}

The datasets used and/or analyzed during the current study are available from the corresponding author on reasonable request.

\section{Competing interests}

The authors declare that they have no competing interests.

\section{Funding}

Faculty of Medicine Siriraj Hospital, Mahidol University, Bangkok, Thailand.

\section{Author contributions}

YK - (1) conception and design, analysis, and interpretation of data; (2) performance of experiments or therapy; (3) drafting of the manuscript or revising it critically for important intellectual content; and (4) final approval of the manuscript submitted.

RT - (1) performance of experiments or therapy; (2) final approval of the manuscript submitted.

TB - (1) conception and design, analysis, and interpretation of data; (2) drafting of the manuscript or revising it critically for important intellectual content; and (3) final approval of the manuscript submitted.

\section{Acknowledgement}

The authors would like to thank Mr. Dittapol Muntham, M.S. (Statistics) for statistical assistance.

\section{References}


1. Garcia MC, Rossen LM, Bastian B, Faul M, Dowling NF, Thomas CC, et al. Potentially Excess Deaths from the Five Leading Causes of Death in Metropolitan and Nonmetropolitan Counties - United States, 2010-2017. MMWR Surveill Summ. 2019;68(10):1-11.

2. Hoffmann MH, Shi H, Schmitz BL, Schmid FT, Lieberknecht M, Schulze R, et al. Noninvasive coronary angiography with multislice computed tomography. JAMA. 2005;293(20):2471-8.

3. Nielsen LH, Botker HE, Sorensen HT, Schmidt M, Pedersen L, Sand NP, et al. Prognostic assessment of stable coronary artery disease as determined by coronary computed tomography angiography: a Danish multicentre cohort study. Eur Heart J. 2017;38(6):413-21.

4. Becker CR, Knez A, Leber A, Hong C, Treede H, Wildhirt S, et al. [Initial experiences with multi-slice detector spiral CT in diagnosis of arteriosclerosis of coronary vessels]. Radiologe. 2000;40(2):118-22.

5. Nieman K, Oudkerk M, Rensing BJ, van Ooijen P, Munne A, van Geuns RJ, et al. Coronary angiography with multi-slice computed tomography. Lancet. 2001;357(9256):599-603.

6. Schroeder S, Kopp AF, Baumbach A, Kuettner A, Georg C, Ohnesorge B, et al. Non-invasive characterisation of coronary lesion morphology by multi-slice computed tomography: a promising new technology for risk stratification of patients with coronary artery disease. Heart. 2001;85(5):5768.

7. Di Leo G, Fisci E, Secchi F, Ali M, Ambrogi F, Sconfienza LM, et al. Diagnostic accuracy of magnetic resonance angiography for detection of coronary artery disease: a systematic review and metaanalysis. Eur Radiol. 2016;26(10):3706-18.

8. Kim WY, Danias PG, Stuber M, Flamm SD, Plein S, Nagel E, et al. Coronary magnetic resonance angiography for the detection of coronary stenoses. N Engl J Med. 2001;345(26):1863-9.

9. Kato S, Kitagawa K, Ishida N, Ishida M, Nagata M, Ichikawa Y, et al. Assessment of coronary artery disease using magnetic resonance coronary angiography: a national multicenter trial. J Am Coll Cardiol. 2010;56(12):983-91.

10. Jin H, Zeng MS, Ge MY, Yun H, Yang S. 3D coronary MR angiography at 1.5 T: Volume-targeted versus whole-heart acquisition. J Magn Reson Imaging. 2013;38(3):594-602.

11. Yoon YE, Kitagawa K, Kato S, Ishida M, Nakajima H, Kurita T, et al. Prognostic value of coronary magnetic resonance angiography for prediction of cardiac events in patients with suspected coronary artery disease. J Am Coll Cardiol. 2012;60(22):2316-22.

12. Mancia G, Fagard R, Narkiewicz K, Redon J, Zanchetti A, Bohm M, et al. 2013 ESH/ESC guidelines for the management of arterial hypertension: The Task Force for the Management of Arterial Hypertension of the European Society of Hypertension (ESH) and of the European Society of Cardiology (ESC). Eur Heart J. 2013;34(28):2159-219.

13. American Diabetes A. Standards of medical care in diabetes--2014. Diabetes Care. 2014;37 Suppl 1: S14-80.

14. Stone NJ, Robinson JG, Lichtenstein AH, Bairey Merz CN, Blum CB, Eckel RH, et al. 2013 ACC/AHA guideline on the treatment of blood cholesterol to reduce atherosclerotic cardiovascular risk in 
adults: a report of the American College of Cardiology/American Heart Association Task Force on Practice Guidelines. J Am Coll Cardiol. 2014;63(25 Pt B):2889-934.

15. Task Force M, Montalescot G, Sechtem U, Achenbach S, Andreotti F, Arden C, et al. 2013 ESC guidelines on the management of stable coronary artery disease: The Task Force on the management of stable coronary artery disease of the European Society of Cardiology. Eur Heart J. 2013;34(38):2949-3003.

16. Krittayaphong R, Chaithiraphan V, Maneesai A, Udompanturak S. Prognostic value of combined magnetic resonance myocardial perfusion imaging and late gadolinium enhancement. Int $\mathrm{J}$ Cardiovasc Imaging. 2011;27(5):705-14.

17. Krittayaphong R, Maneesai A, Saiviroonporn P, Nakyen S, Thanapiboonpol P, Yindeengam A. Prevalence and characters of anomalous coronary artery from coronary magnetic resonance angiography. J Med Assoc Thai. 2014;97 Suppl 3: S124-31.

18. Fischer SE, Wickline SA, Lorenz $\mathrm{CH}$. Novel real-time R-wave detection algorithm based on the vectorcardiogram for accurate gated magnetic resonance acquisitions. Magn Reson Med. 1999;42(2):361-70.

19. Stuber M, Botnar RM, Danias PG, Kissinger KV, Manning WJ. Submillimeter three-dimensional coronary MR angiography with real-time navigator correction: comparison of navigator locations. Radiology. 1999;212(2):579-87.

20. Wu YW, Tadamura E, Yamamuro M, Kanao S, Nakayama K, Togashi K. Evaluation of threedimensional navigator-gated whole heart MR coronary angiography: the importance of systolic imaging in subjects with high heart rates. Eur J Radiol. 2007;61(1):91-6.

21. Cerqueira MD, Weissman NJ, Dilsizian V, Jacobs AK, Kaul S, Laskey WK, et al. Standardized myocardial segmentation and nomenclature for tomographic imaging of the heart. A statement for healthcare professionals from the Cardiac Imaging Committee of the Council on Clinical Cardiology of the American Heart Association. Circulation. 2002;105(4):539-42.

22. Austen WG, Edwards JE, Frye RL, et al. A reporting system on patients evaluated for coronary artery disease. Report of the Ad Hoc Committee for Grading of Coronary Artery Disease, Council on Cardiovascular Surgery, American Heart Association. Circulation 1975;51 Suppl 4:5-40.

23. Hicks KA, Mahaffey KW, Mehran R, Nissen SE, Wiviott SD, Dunn B, et al. 2017 Cardiovascular and Stroke Endpoint Definitions for Clinical Trials. Circulation. 2018;137(9):961-72.

24. Jahnke C, Nagel E, Gebker R, et al. Prognostic value of cardiac magnetic resonance stress tests: adenosine stress perfusion and dobutamine stress wall motion imaging. Circulation. 2007; 115 :1769-1776.

25. Lipinski MJ, McVey CM, Berger JS, Kramer CM, Salerno M. Prognostic value of stress cardiac magnetic resonance imaging in patients with known or suspected coronary artery disease: a systematic review and meta-analysis. J Am Coll Cardiol. 2013;62(9):826-38.

26. American College of R, Society of Cardiovascular Computed T, Society for Cardiovascular Magnetic R, American Society of Nuclear C, North American Society for Cardiac I, Society for Cardiovascular A, 
et al. ACCF/ACR/SCCT/SCMR/ASNC/NASCI/SCAI/SIR 2006 appropriateness criteria for cardiac computed tomography and cardiac magnetic resonance imaging. A report of the American College of Cardiology Foundation Quality Strategic Directions Committee Appropriateness Criteria Working Group. J Am Coll Radiol. 2006;3(10):751-71.

27. Hajhosseiny R, Bustin A, Munoz C, Rashid I, Cruz G, Manning WJ, et al. Coronary Magnetic Resonance Angiography: Technical Innovations Leading Us to the Promised Land? JACC Cardiovasc Imaging. 2020;13(12):2653-72.

28. Hamdy A, Ishida M, Sakuma H. Cardiac MR Assessment of Coronary Arteries. Cardiovascular Imaging Asia. 2017;1(1):49-59.

29. Spuentrup E, Katoh M, Buecker A, Manning WJ, Schaeffter T, Nguyen TH, et al. Free-breathing 3D steady-state free precession coronary MR angiography with radial k-space sampling: comparison with cartesian k-space sampling and cartesian gradient-echo coronary MR angiography-pilot study. Radiology. 2004;231(2):581-6.

30. Krittayaphong R, Mahanonda N, Kangkagate C, Nakyen S, Tanapibunpon P, Chaithiraphan S. Accuracy of magnetic resonance imaging in the diagnosis of coronary artery disease. $\mathrm{J}$ Med Assoc Thai. 2003;86 Suppl 1: S59-66.

31. Langer $\mathrm{C}$, Peterschroder A, Franzke K, Esdorn $\mathrm{H}$, Korperich $\mathrm{H}$, Meyer $\mathrm{H}$, et al. Noninvasive coronary angiography focusing on calcification: multislice computed tomography compared with magnetic resonance imaging. J Comput Assist Tomogr. 2009;33(2):179-85.

\section{Tables}

Table 1. Baseline Characteristics of Patients with and without Significant Coronary Artery Stenosis. 


\begin{tabular}{|c|c|c|c|c|}
\hline & $\begin{array}{l}\text { Total } \\
(n=389)\end{array}$ & $\begin{array}{l}\text { Significant } \\
\text { Stenosis } \\
(n=139)\end{array}$ & $\begin{array}{l}\text { No Significant } \\
\text { Stenosis } \\
(n=250)\end{array}$ & $\begin{array}{l}\text { p- } \\
\text { Value }\end{array}$ \\
\hline Male & $187(48)$ & $76(55)$ & $111(44)$ & 0.05 \\
\hline Age, years & $\begin{array}{l}68.1 \pm \\
10.8\end{array}$ & $69.4 \pm 9.9$ & $67.3 \pm 11.3$ & 0.07 \\
\hline Systolic blood pressure, $\mathrm{mmHg}$ & $\begin{array}{l}136.5 \pm \\
18.9\end{array}$ & $139.9 \pm 18.7$ & $134.7 \pm 18.9$ & 0.008 \\
\hline Diastolic blood pressure, $\mathrm{mmHg}$ & $\begin{array}{l}73.8 \pm \\
11.7\end{array}$ & $72.6 \pm 12.3$ & $74.4 \pm 11.4$ & 0.16 \\
\hline Heart rate, beats/minutes & $\begin{array}{l}77.5 \pm \\
13.6\end{array}$ & $76.1 \pm 14.1$ & $78.2 \pm 13.3$ & 0.15 \\
\hline \multicolumn{5}{|l|}{ Clinical history } \\
\hline Hypertension & $340(87)$ & $126(91)$ & $214(86)$ & 0.15 \\
\hline Diabetes mellitus & $215(55)$ & $81(58)$ & $134(54)$ & 0.37 \\
\hline Hyperlipidemia & $292(75)$ & $114(82)$ & $178(71)$ & 0.02 \\
\hline Stable coronary artery disease & $61(16)$ & $45(32)$ & $16(6)$ & $<0.001$ \\
\hline Previous myocardial infarction & $12(3)$ & $11(8)$ & $1(0.4)$ & $<0.001$ \\
\hline Stroke & $17(4)$ & $5(4)$ & $12(5)$ & 0.58 \\
\hline Current smoking & $70(18)$ & $40(29)$ & $30(12)$ & $<0.001$ \\
\hline Chest pain & $133(34)$ & $39(28)$ & $94(38)$ & 0.06 \\
\hline Dyspnea & $62(16)$ & $18(13)$ & $44(18)$ & 0.23 \\
\hline Heart failure & $33(8)$ & $13(9)$ & $20(8)$ & 0.65 \\
\hline \multicolumn{5}{|l|}{ Medications } \\
\hline ACEI or ARB & $171(44)$ & $63(45)$ & $108(43)$ & 0.69 \\
\hline Antiplatelet & $184(47)$ & $78(56)$ & $106(42)$ & 0.01 \\
\hline Beta blocker & $186(48)$ & $67(48)$ & $119(48)$ & 0.91 \\
\hline Calcium channel blocker & $123(32)$ & $41(30)$ & $82(33)$ & 0.50 \\
\hline Nitrate & $54(14)$ & $34(25)$ & $20(8)$ & $<0.001$ \\
\hline Statin & $196(50)$ & $81(58)$ & $115(46)$ & 0.02 \\
\hline \multicolumn{5}{|l|}{ CMR } \\
\hline LVEDV index, $\mathrm{ml} / \mathrm{m}^{2}$ & $47.3 \pm$ & $59.6 \pm 47.0$ & $40.4 \pm 38.0$ & $<0.001$ \\
\hline
\end{tabular}


42.4

\begin{tabular}{|lllll|}
\hline LVESV index, $\mathrm{ml} / \mathrm{m}^{2}$ & $27.8 \pm$ & $34.9 \pm 28.1$ & $23.8 \pm 22.0$ & $<0.001$ \\
\hline LVEF, \% & 24.9 & & & $<0.001$ \\
\hline Late gadolinium enhancement & $14.1 \pm$ & $63.1 \pm 16.4$ & $69.8 \pm 12.1$ & $<0.001$ \\
\hline $\begin{array}{l}\text { Number of coronary artery } \\
\text { stenosis }\end{array}$ & $81(21)$ & $64(46)$ & $17(7)$ & - \\
\hline 1-vessel & $66(17)$ & $66(48)$ & - & - \\
\hline 2-vessel & $49(13)$ & $49(35)$ & - & - \\
\hline 3-vessel & $24(6)$ & $24(17)$ & - & \\
\hline
\end{tabular}

Values are numbers (percentages) or mean \pm standard deviation. Bold values are $<0.05$.

$\mathrm{ACEI}$ = angiotensin-converting enzyme inhibitor; $\mathrm{ARB}$ = angiotensin II receptor blocker; $\mathrm{CMR}=$ cardiac magnetic resonance; LVEDV = left ventricular end diastolic volume; LVEF = left ventricular ejection fraction; LVESV = left ventricular end systolic volume.

Table 2. Cardiac Events.

\begin{tabular}{|c|c|c|c|c|c|}
\hline & $\begin{array}{l}\text { Total } \\
(n=389)\end{array}$ & $\begin{array}{l}\text { Significant } \\
\text { Stenosis } \\
(n=139)\end{array}$ & $\begin{array}{l}\text { No Significant } \\
\text { Stenosis } \\
(n=250)\end{array}$ & $\mathrm{HR}(95 \% \mathrm{Cl})$ & $\begin{array}{l}\mathrm{p}- \\
\text { Value }\end{array}$ \\
\hline Cardiac mortality & $8(2.1)$ & $6(4.3)$ & $2(0.8)$ & \multirow{2}{*}{$\begin{array}{l}5.57(1.12- \\
27.61)\end{array}$} & 0.04 \\
\hline Non-fatal myocardial & $19(4.9)$ & 14 (10.1) & $5(2.0)$ & & 0.001 \\
\hline \multirow{2}{*}{$\begin{array}{l}\text { Hospitalization for heart } \\
\text { failure }\end{array}$} & $34(8.7)$ & $19(13.7)$ & $15(6.0)$ & $\begin{array}{l}5.44(1.96- \\
15.13)\end{array}$ & 0.01 \\
\hline & $10(2.6)$ & $9(6.5)$ & $1(0.4)$ & $\begin{array}{l}2.43(1.24- \\
4.79)\end{array}$ & 0.01 \\
\hline Late revascularization & & & & $\begin{array}{l}17.58(2.23- \\
138.85)\end{array}$ & \\
\hline
\end{tabular}

Values are numbers (percentages). Bold values are $<0.05$.

$\mathrm{Cl}=$ confidence interval; $\mathrm{HR}=$ hazard ratio .

Table 3. Univariate and Multivariate Analyses of Hard Cardiac Events. 


\begin{tabular}{|c|c|c|c|c|}
\hline & \multicolumn{2}{|l|}{ Univariate Analysis } & \multicolumn{2}{|c|}{ Multivariate Analysis } \\
\hline & $\mathrm{HR}(95 \% \mathrm{Cl})$ & $\mathrm{p}$-Value & $\mathrm{HR}(95 \% \mathrm{Cl})$ & p-Value \\
\hline Male & $1.03(0.45-2.34)$ & 0.93 & & \\
\hline Age, years & $1.01(0.96-1.04)$ & 0.69 & & \\
\hline Systolic blood pressure, per $1 \mathrm{mmHg}$ & $1.001(0.97-1.02)$ & 0.90 & & \\
\hline Diastolic blood pressure, per $1 \mathrm{mmHg}$ & $0.98(0.95-1.02)$ & 0.56 & & \\
\hline Heart rate, beats/minutes & $1.01(0.98-1.04)$ & 0.51 & & \\
\hline Hypertension & $1.64(0.38-6.99)$ & 0.50 & & \\
\hline Diabetes mellitus & $1.09(0.48-2.50)$ & 0.82 & & \\
\hline Hyperlipidemia & $0.82(0.32-2.09)$ & 0.69 & & \\
\hline Stable coronary artery disease & $2.10(0.82-5.34)$ & 0.12 & & \\
\hline Previous myocardial infarction & $4.49(1.33-15.11)$ & 0.02 & $1.43(0.37-5.59)$ & 0.61 \\
\hline Current smoking & $1.21(0.45-3.27)$ & 0.70 & & \\
\hline Chest pain & $1.54(0.67-3.50)$ & 0.30 & & \\
\hline Dyspnea & $1.28(0.47-3.44)$ & 0.63 & & \\
\hline Heart failure & $2.81(1.04-7.59)$ & 0.04 & - & - \\
\hline ACEI or ARB & $1.23(0.54-2.80)$ & 0.61 & & \\
\hline Antiplatelet & $1.65(0.71-3.82)$ & 0.24 & & \\
\hline Beta blocker & $1.33(0.58-3.05)$ & 0.49 & & \\
\hline Calcium channel blocker & $1.18(0.50-2.80)$ & 0.69 & & \\
\hline Nitrate & $2.91(1.23-6.88)$ & 0.02 & $1.34(0.50-3.59)$ & 0.57 \\
\hline Statin & $1.34(0.59-3.07)$ & 0.48 & & \\
\hline LVEDV index, $\mathrm{ml} / \mathrm{m}^{2}$ & $1.01(1.001-1.01)$ & 0.01 & - & - \\
\hline LVESV index, $\mathrm{ml} / \mathrm{m}^{2}$ & $1.01(1.004-1.02)$ & 0.003 & - & - \\
\hline LVEF, \% & $0.96(0.94-0.98)$ & 0.004 & $0.99(0.96-1.02)$ & 0.50 \\
\hline Late gadolinium enhancement & $4.75(2.09-10.79)$ & $<0.001$ & $1.78(0.57-5.56)$ & 0.32 \\
\hline Significant coronary artery stenosis & $5.52(2.17-14.01)$ & $<0.001$ & $3.35(1.13-9.96)$ & 0.03 \\
\hline
\end{tabular}

Bold values are $<0.05$. 
$\mathrm{ACEl}$ = angiotensin-converting enzyme inhibitor; $\mathrm{ARB}=$ angiotensin II receptor blocker; $\mathrm{Cl}=$ confidence interval; $\mathrm{CMR}$ = cardiac magnetic resonance; $\mathrm{HR}$ = hazard ratio; LVEDV = left ventricular end diastolic volume; LVEF = left ventricular ejection fraction; LVESV = left ventricular end systolic volume.

Table 4. Univariate and Multivariate Analyses of MACE. 


\begin{tabular}{|c|c|c|c|c|}
\hline & \multicolumn{2}{|l|}{ Univariate Analysis } & \multicolumn{2}{|c|}{ Multivariate Analysis } \\
\hline & $\mathrm{HR}(95 \% \mathrm{Cl})$ & $\mathrm{p}$-Value & $\mathrm{HR}(95 \% \mathrm{Cl})$ & p-Value \\
\hline Male & $1.22(0.70-2.10)$ & 0.47 & & \\
\hline Age, years & $1.02(0.99-1.04)$ & 0.12 & & \\
\hline Systolic blood pressure, per $1 \mathrm{mmHg}$ & $1.00(0.98-1.01)$ & 0.99 & & \\
\hline Diastolic blood pressure, per $1 \mathrm{mmHg}$ & $0.97(0.95-1.01)$ & 0.01 & & \\
\hline Heart rate, beats/minutes & $1.01(0.98-1.02)$ & 0.66 & & \\
\hline Hypertension & $1.46(0.58-3.68)$ & 0.42 & & \\
\hline Diabetes mellitus & $1.03(0.59-1.78)$ & 0.91 & & \\
\hline Hyperlipidemia & $1.11(0.57-2.16)$ & 0.76 & & \\
\hline Stable coronary artery disease & $2.09(1.11-3.92)$ & 0.02 & - & - \\
\hline Previous myocardial infarction & $3.87(1.53-9.74)$ & 0.004 & $1.70(0.66-4.35)$ & 0.27 \\
\hline Current smoking & $1.84(1.01-3.35)$ & 0.04 & - & - \\
\hline Chest pain & $1.36(0.78-2.35)$ & 0.27 & & \\
\hline Dyspnea & $1.11(0.56-2.22)$ & 0.76 & & \\
\hline Heart failure & $4.59(2.52-8.37)$ & $<0.001$ & $3.28(1.62-6.63)$ & 0.001 \\
\hline ACEI or ARB & $1.34(0.78-2.31)$ & 0.28 & & \\
\hline Antiplatelet & $1.14(0.66-1.97)$ & 0.62 & & \\
\hline Beta blocker & $1.02(0.59-1.75)$ & 0.94 & & \\
\hline Calcium channel blocker & $0.64(0.33-1.22)$ & 0.18 & & \\
\hline Nitrate & $1.89(1.01-3.55)$ & 0.04 & & \\
\hline Statin & $1.11(0.64-1.91)$ & 0.70 & & \\
\hline LVEDV index, $\mathrm{ml} / \mathrm{m}^{2}$ & $1.01(1.005-1.01)$ & $<0.001$ & - & - \\
\hline LVESV index, $\mathrm{ml} / \mathrm{m}^{2}$ & $1.02(1.01-1.02)$ & $<0.001$ & - & - \\
\hline LVEF, \% & $0.96(0.94-0.97)$ & $<0.001$ & $0.99(0.97-1.01)$ & 0.39 \\
\hline Late gadolinium enhancement & $4.62(2.68-7.98)$ & $<0.001$ & $2.27(1.05-4.91)$ & 0.03 \\
\hline Significant coronary artery stenosis & $3.49(1.98-6.14)$ & $<0.001$ & $2.00(1.02-3.90)$ & 0.04 \\
\hline
\end{tabular}

Bold values are $<0.05$. 
$\mathrm{ACEI}=$ angiotensin-converting enzyme inhibitor; $\mathrm{ARB}=$ angiotensin II receptor blocker; $\mathrm{Cl}=$ confidence interval; $\mathrm{CMR}$ = cardiac magnetic resonance; $\mathrm{HR}$ = hazard ratio; $\mathrm{LVEDV}=$ left ventricular end diastolic volume; LVEF = left ventricular ejection fraction; LVESV = left ventricular end systolic volume; $\mathrm{MACE}=$ major adverse cardiac events.

\section{Figures}

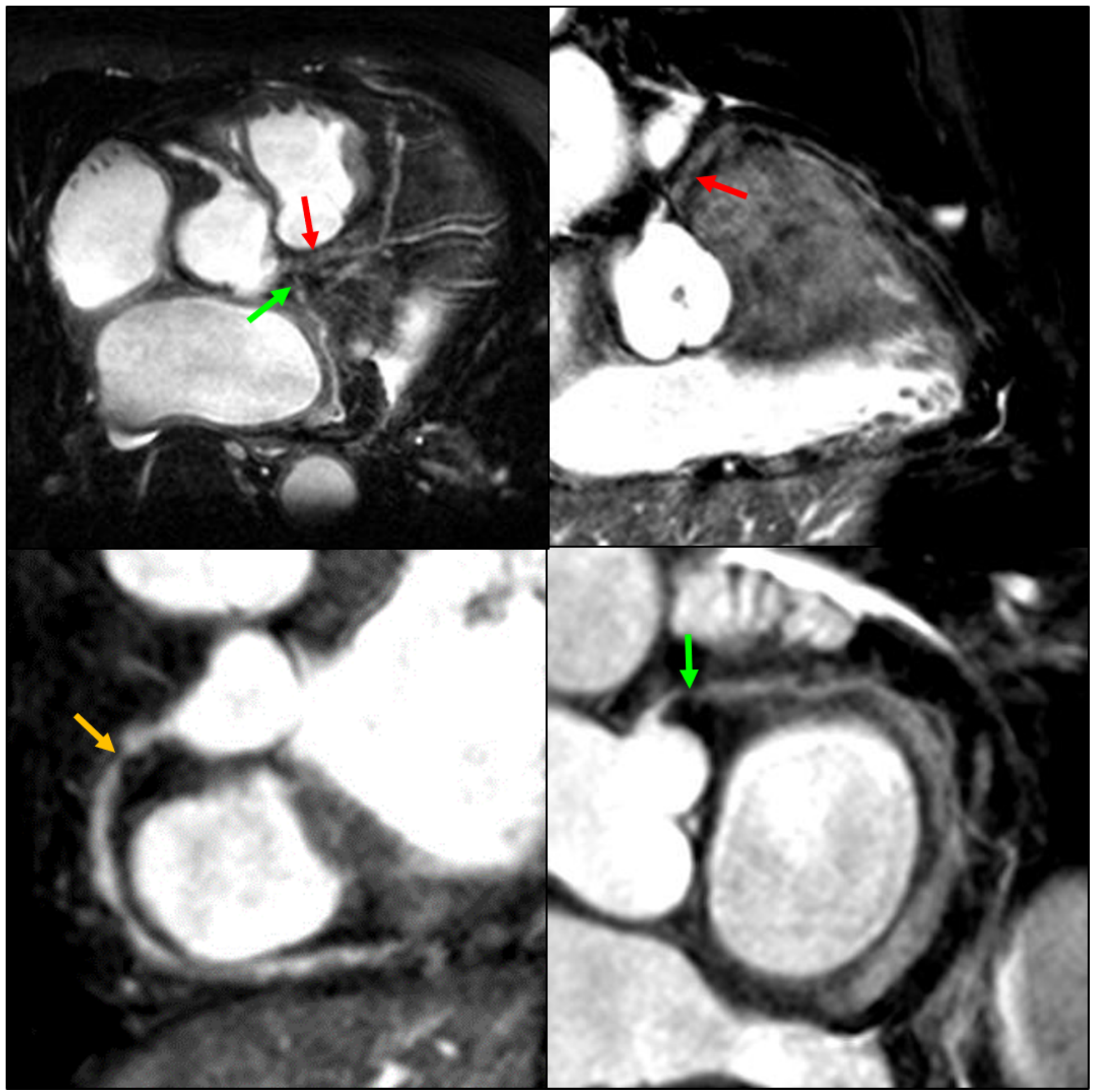

Figure 1 
Images illustrating MRCA findings in a 62-year-old man presenting with chest pain. Maximum intensity projection images showed significant stenosis in the proximal part of the left anterior descending artery (red arrow), left circumflex artery (green arrow), and right coronary artery (yellow arrow). MRCA = magnetic resonance coronary angiography.

A

Hard Cardiac Events

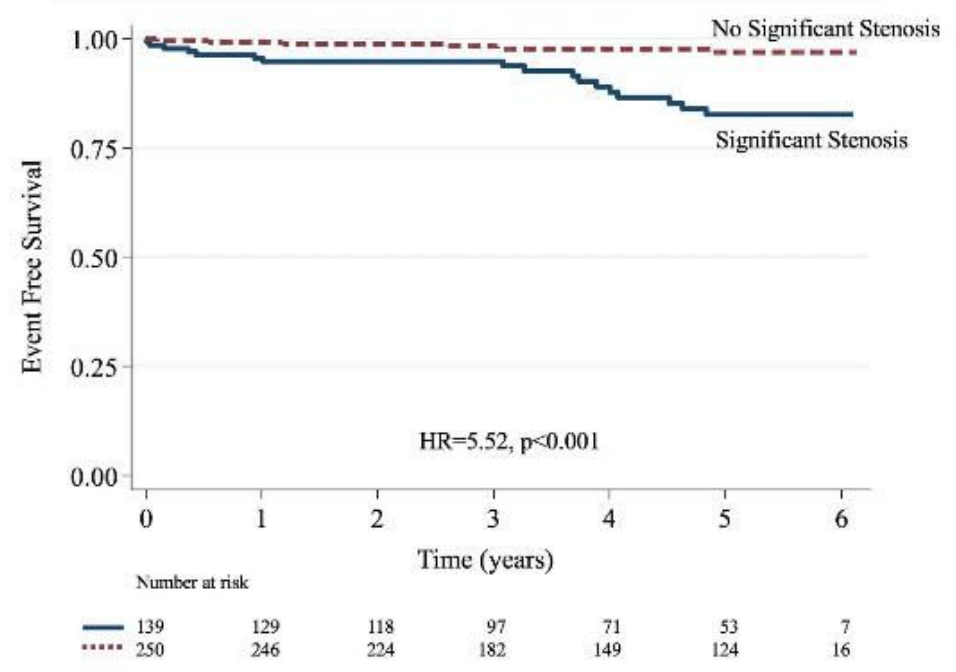

B

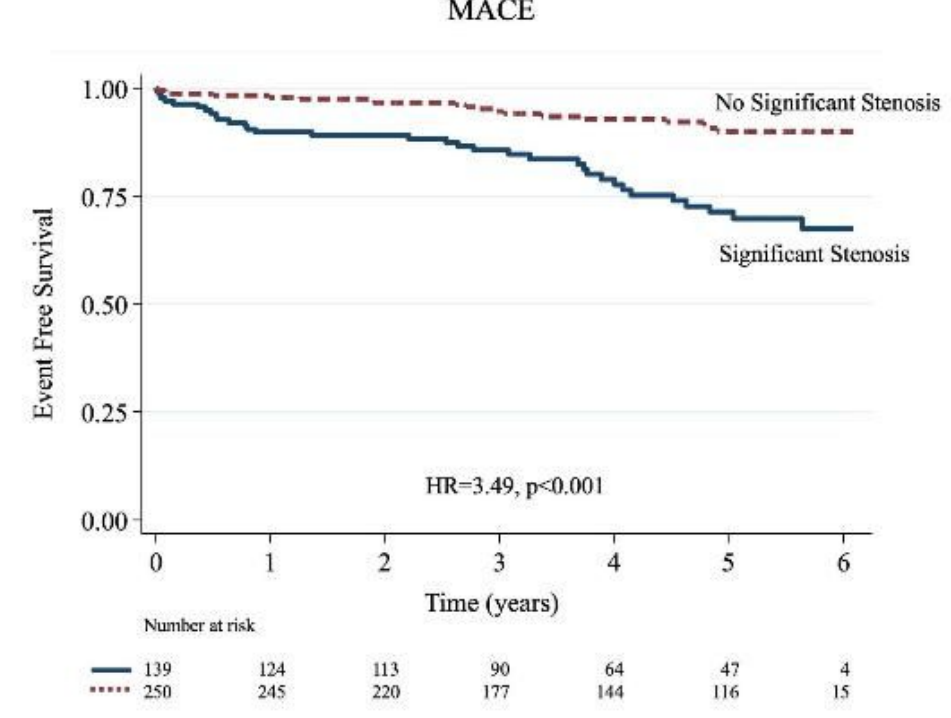

Figure 2

Kaplan-Meier survival curves for hard cardiac events (A) and MACE (B). MACE = major adverse cardiac events.

A

Hard Cardiac Events

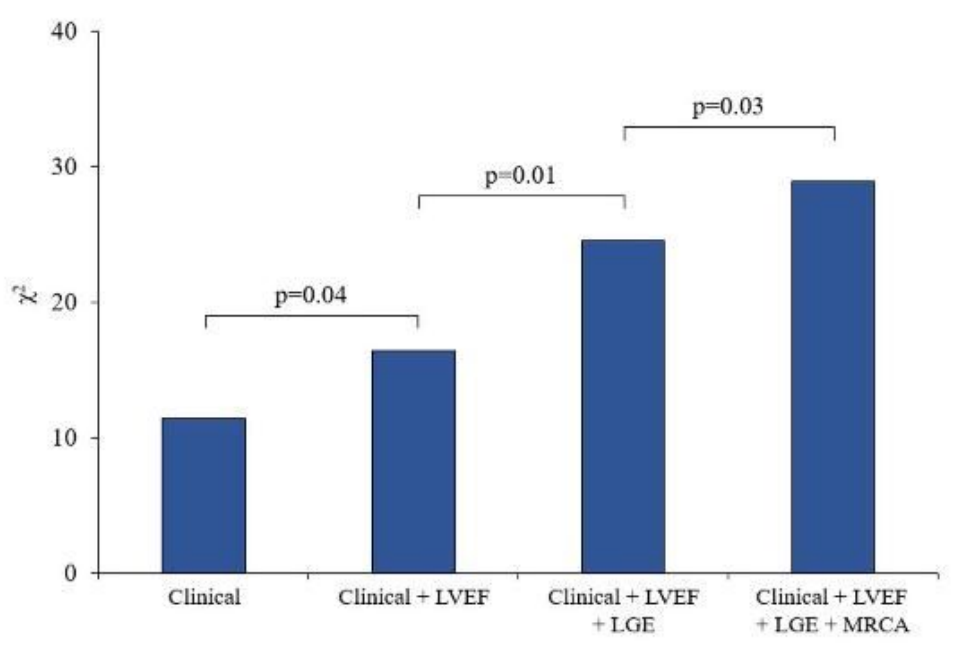

B

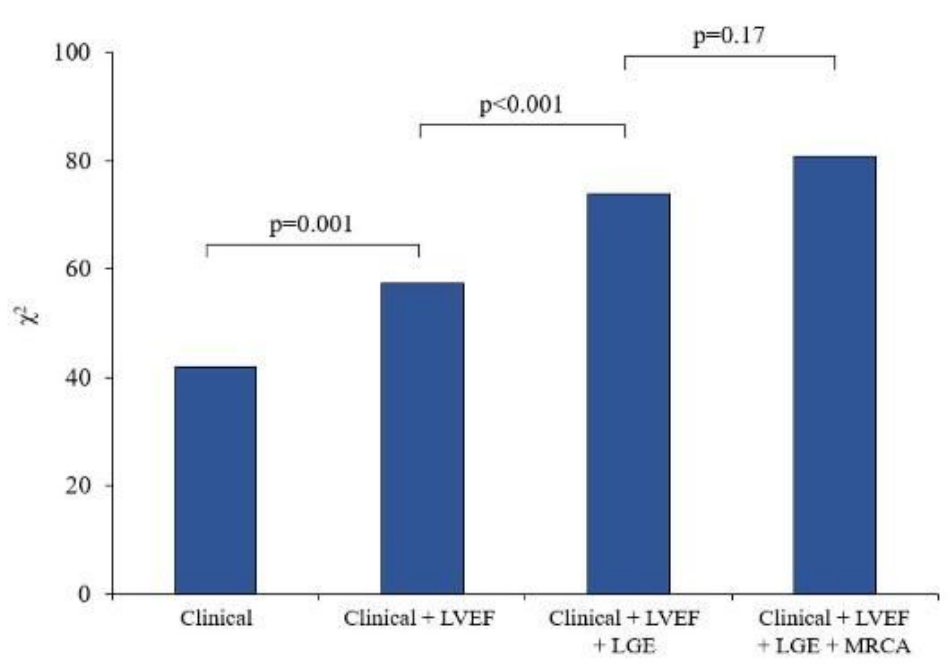

\section{Figure 3}

Incremental prognostic value of LVEF, LGE, and MRCA for the prediction of hard cardiac events $(A)$ and MACE (B). Clinical = age, male gender, history of heart failure, and previous myocardial infarction. $L G E=$ 
late gadolinium enhancement; LVEF = left ventricular ejection fraction; $\mathrm{MACE}=$ major adverse cardiovascular events; MRCA = magnetic resonance coronary angiography.

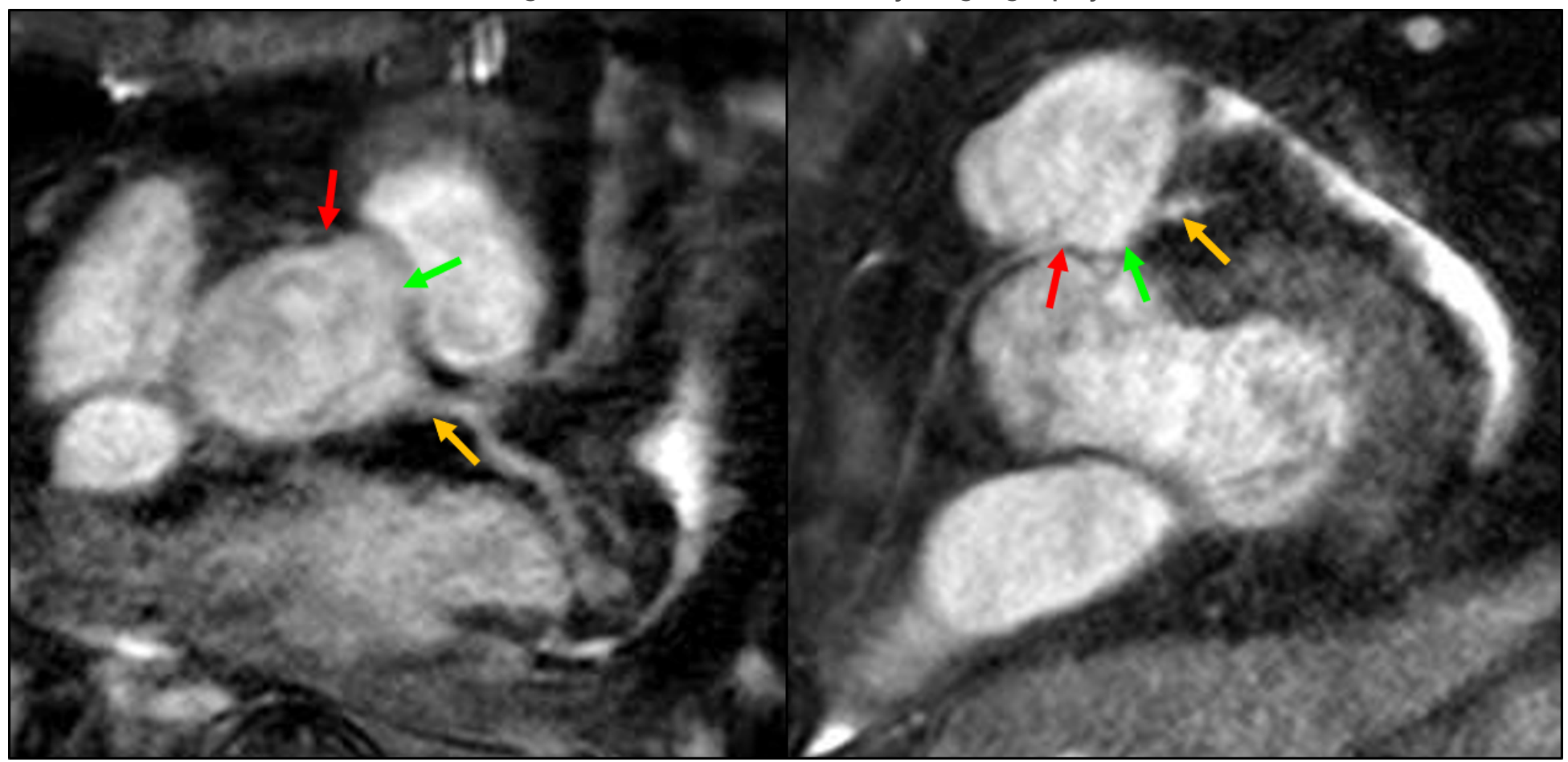

Figure 4

MRCA images showing a malignant-type anomalous coronary artery in a 40-year-old man with chest pain during exercise (right coronary artery ostium-red arrow, left main coronary artery-yellow arrow, and left coronary cusp of the aortic valve-green arrow). MRCA = magnetic resonance coronary angiography. 